\title{
A Wavelet-Based Method to Measure Stock Market Development
}

\author{
Adel Al Sharkasi ${ }^{1}$, Heather J. Ruskin ${ }^{2}$, Martin Crane ${ }^{2}$, José Matos ${ }^{3}$, Sílvio M. A. Gama ${ }^{4}$ \\ ${ }^{1}$ Department of Statistics, Faculty of Science, University of Benghazi, Benghazi, Libya \\ ${ }^{2}$ School of Computing, Dublin City University, Dublin, Ireland \\ ${ }^{3}$ CMUP \& Grupo de Matemática Informática, Faculdade de Econmia, Universidade do Porto, Porto, Portugal \\ ${ }^{4}$ CMUP \& Departamento de Matemática Aplicada, Faculdade de Ciencias, Universidade do Porto, Porto, Portugal \\ Email: adelsharkasi@yahoo.com, hruskin@computing.dcu.ie, mcrane@computing.dcu.ie, \\ jamatos@fc.up.pt, smgama@fc.up.pt
}

Received November 19, 2013; revised December 19, 2013; accepted December 27, 2013

Copyright (C 2014 Adel Al Sharkasi et al. This is an open access article distributed under the Creative Commons Attribution License, which permits unrestricted use, distribution, and reproduction in any medium, provided the original work is properly cited. In accordance of the Creative Commons Attribution License all Copyrights @ 2014 are reserved for SCIRP and the owner of the intellectual property Adel Al Sharkasi et al. All Copyright (C) 2014 are guarded by law and by SCIRP as a guardian.

\begin{abstract}
In this paper, we introduce a novel algorithm, based on the wavelet transform, to measure stock market development. This algorithm is applied to the return series of fourteen worldwide market indices from 1996 to 2005. We find that a comparison of the return series in terms of the quantity of fractional Gaussian noise (fGn), for different values of Hurst exponent $(H)$, facilitates the classification of stock markets according to their degree of development. We also observe that the simple classification of stock markets into "emerging" or "developing" and "mature" or "developed" is no longer sufficient. However, stock markets can be grouped into three categories that we named emerging, intermediate and mature.
\end{abstract}

\section{KEYWORDS}

\section{Wavelet Transform; Hurst Exponent (H); Stock Market Classifications}

\section{Introduction}

There is no precise set of criteria which clearly distinguishes between different stock market types. Therefore, different institutions use different criteria to group countries (or stock markets) by their development level. The World Bank, for example, classifies stock markets into emerging and mature, depending on their national economies using GNP per capita ${ }^{1}$. This classification is, however, disputable for several reasons not least the fact that most developed countries are still undergoing development and some countries, still considered as "developing", have graduated to a further stage over time.

In relation to ways in which stock market development can be measured, there is neither a common concept nor a common indicator agreed by Economists. For example, Demirguc-Kunt and Levine [1] compared many different developmental measures, including market size, liquidity, concentration, volatility, institutional development and international integration, across forty-one countries. Their findings indicated that: 1) small stock markets are less liquid, more volatile and less internationally integrated than larger markets. 2) Richer countries generally are more developed than poorer ones. Exceptions include some stock markets defined as "developing" on the basis of national economy, (e.g. those of the Republic of Korea, Malaysia and Thailand). These show indications of maturity stronger than many "mature" markets (e.g. those of Australia, Canada and many European countries).

Recently, Di Matteo et al. ([2] and [3]) studied the scaling properties of different global stock market indices

${ }^{1}$ The World Bank refer to low-income (GNP per capita of $\$ 765$ or less) and middle-income (GNP of $\$ 9,385$ or less) countries as “developing and to high-income countries ( $\$ 9,386$ or more) as “developed”. 
by using the generalized Hurst approach. They found in particular that a) Deviations from pure Brownian motion behavior are associated with the degree of the market's development. b) The generalized Hurst exponent $(H)$ is a powerful tool in distinguishing between the degree of development of stock markets with emerging and mature markets having $H>0.5$ and $H<0.5$ respectively.

In [4], we have attempted to differentiate, qualitatively, between emerging and mature markets using Principle Component Analysis. In particular, we investigated the behaviour of the first three eigenvalues of the covariance matrices of the return series $\left(\lambda_{1}, \lambda_{2}, \lambda_{3}\right)$ and the ratios using the wavelet transform and eigenvalue analysis. This, in order to study the reaction of emerging and mature markets to crashes and events, and also to measure the recovery time for these two market types. Our results indicated that mature markets respond to crashes differently to emerging ones, in that emerging markets may take up to two months to recover while major markets take less than a month to do so. In addition, the results showed that the subdominant eigenvalues $\left(\lambda_{2}, \lambda_{3}\right)$ give additional information on market movement, especially for emerging markets and that a study of the behavior of the other eigenvalues may provide insight on crash dynamics. We also found that emerging markets show evidence of persistent behaviour, while mature markets exhibit anti-persistent behaviour (see also [5]).

In our most recent work [5], we suggest a new method based on a time-scale extension of Detrended Fluctuation Analysis (TSDFA) in order to study the behaviour of different stock markets in different time periods and at different scale points. Our results implied that there are three groups of stock market: 1) Very mature markets, e.g. UK, US and Japan, which behave in a mature manner for all time and scale points. 2) Emerging markets, e.g. India, Egypt and Sir Lanka, which act as emerging at all time and scale points. 3) Other stock markets, e.g. Canada, which has behaviour which is varied depending on the time period and scale (i.e. emergent for some, mature for others).

Since the Hurst exponent $(H)$ is very sensitive to the stock market stage of development, we, therefore, suggest a new algorithm, based on the discrete wavelet transform (DWT) and fractional Gaussian noise (fGn) assessed for different values of $H$, and evaluate its performance here. Investors are interested in knowing market type in order to make the right investment decisions; several studies notably (e.g. [4,6-9]) have reported that emerging markets consistently behave differently to mature ones. Moreover, for foreign investors, emerging markets are more attractive because of their investment opportunities for making higher returns. However, they are riskier and more volatile due to some of their structural issues, such as foreign debt and political instability, while mature markets are safer, more solid and more stable. Therefore, the investors' goal is to find a risk-return balance which generates some returns (or profit) with acceptable risk.

The remainder of this paper is organized as follows: the methodology used in this study is described briefly in Section 2, with data and results given in Section 3. The final section provides a discussion of the results and gives principal conclusions.

\section{Methodology}

\subsection{Fractional Gaussian Noise}

Fractional Gaussian noise ( $\mathrm{fGn}$ ) series $\left\{X_{i}, i \geq 1\right\}$ is a self-similar process that is indexed by the Hurst exponent $H$ (where $0<H<1$.) It is the increment of fractional Brownian motion $(\mathrm{fBm})^{2}$, namely

$$
X_{i}=f B m_{H}(i+1)-f B m_{H}(i), i \geq 1
$$

with zero mean, where the auto-covariance function $\gamma(t)=E\left(X_{i} X_{i+t}\right)$ is given by

$$
\gamma(t)=2^{-1}\left[(t+1)^{2 H}-2 t^{2 H}+|t-1|^{2 H}\right], t \geq 0
$$

An important point about $\gamma$ is that it satisfies

$$
\gamma(t) \sim H(2 H-1) t^{2 H-2} \text { as } t \rightarrow \infty
$$

For $H<0.5$, fractional Gaussian noise (fGn) demonstrates anti-persistent behaviour, where this implies that if a series is down in one period then it is more likely to rebound in the next period. For fGn with $H>0.5$, long memory or persistent behaviour is demonstrated. If, e.g., a series is down in a given period, then it is likely that in the next period this behaviour will be sustained. The special case of fGn with $H=0.5$ corresponds to Gaussian white noise, representing randomness and implying that the values are uncorrelated. Fractional Gaussian

${ }^{2}$ It exhibits complex but linear long-term dependencies and is characterized by Hurst exponent $(H \in[0,1])$. 
noise series (fGn), corresponding to different values of $H(0.3,0.4,0.5,0.6$ and 0.7$)$, have been simulated here using the S-plus function ${ }^{3}$ in order to compare behaviour with that of the return series of stock market indices.

\subsection{Our Wavelet-Based Classification}

Several studies (such as: [2-4]) conclude that developing and developed markets exhibit persistent $(H>0.5)$ and anti-persistent $(H<0.5)$ behaviour respectively, indicating that the development of a stock market is associated with the change in its behaviour from persistence to anti-persistence. Based on this, we have developed an extended DWT technique, which is described by the following steps:

1) We simulate a set of one hundred series of 3000 (each) points of fractional Gaussian noise (fGn) with each $H \in\{0.3,0.4,0.5,0.6,0.7\}$.

2) For each set, we apply the DWT in order to compute the energy percentage explained by each wavelet component for the 100 series in each set and take the average of these percentages (Table 1).

3) We estimate the energy percentage explained by each wavelet component for the return series of stock market indices (Table 1).

4) The logarithm to base two of the energy percentages ${ }^{4},\left(\log _{2}\right.$ (energy\%)), explained by the first six components $\left(d_{1}-d_{6}\right)$, are calculated and plotted in Figure 1.

5) The behaviour of the return series (or that of the linear fit of the returns) is compared with that of the fGn series, for different values of $H$, in order to group the stock markets.

\section{Data and Results}

\subsection{Data Description}

Our empirical analysis is performed on the daily returns of fourteen worldwide market indices which are listed in Table 2, where Daily Return $=\ln \left(P_{t} / P_{t-1}\right)$, where $P_{t}$ and $P_{t-1}$ are the closing price of the index at day $t$ and $t-$ 1 respectively.

The World Bank classification of stock markets is given in Table 3, with the two basic groups consisting of:

- Emerging: Argentina, Czech Republic, Ireland, Mexico, Portugal, Russia and Singapore.

- Mature: Australia, Canada, Germany, Hong Kong, Japan, the UK and the US.

The energy percentages described by each wavelet component for the daily returns of the fourteen market indices are given also in Table 1 . This shows that the first two high frequency components $\left(d_{1}\right.$ and $\left.d_{2}\right)$ explain more than $65 \%$ of the energy of these series, implying that movements are mainly caused by short-term fluctuations.

\subsection{Empirical Results}

The algorithm of Section 2.2, which is designed to measure the degree of development of a stock market, has been applied to fourteen global stock markets listed in Table 1. The results are given in Figure 1. Firstly, we need to clarify the following key points:

- Developing and Developed markets demonstrate persistent and anti-persistent behaviour respectively (with correspondingly, $H>0.5$ and $H<0.5$ ). The expectation, therefore, is that the stock market should move from persistence to anti-persistence as it develops.

- Our new approach allows for variation in $\boldsymbol{H}$, but a market will fall on one side or another of the well-defined threshold of $H=0.5$ (Gaussian noise) when it is exhibiting clear persistent or anti-persistent behaviour. Note that these are fixed values of $H=H(\tau, \theta)$ with $\tau=$ length of series (number of observations) and $\theta=$ number of trading days $=1$.

- The behaviour of the linear fit of logarithms of stock market returns is compared with that of the generated fractional Gaussian noise (fGn) series for different values of $H$. The straight line fit for the fGn log series versus the wavelet components indicates that the $d_{1}$ doublet explains the largest percentage of energy, $d_{2}$ the next largest and so on.

For classification purposes, we compare the behaviour of the linear fit ${ }^{5}$ of the returns of stock market indices to that of the fractional Gaussian noise (fGn) series with different values of $H$. From Figure 1, we can see that:

\footnotetext{
${ }^{3}$ Simulate. FARIMA $(0, d, 0)$, where $d=H-0.5$.

${ }^{4}$ Logarithm to base two is used because there are $2^{j}$ coefficients in the $j^{\text {th }}$ wavelet component, where $j=1,2, \cdots, 6$.

${ }^{5}$ We estimated the linear model for the return series because we want to have clearer comparison where the logarithms of all fGn are linear lines.
} 
Table 1. Percentages of energy explained by wavelet components for the daily returns of indices series.

\begin{tabular}{cccccccc}
\hline $\begin{array}{c}\text { W. component } \rightarrow \\
\text { market } \downarrow\end{array}$ & $d_{1}$ & $d_{2}$ & $d_{3}$ & $d_{4}$ & $d_{5}$ & $d_{6}$ & $S_{6}$ \\
\hline Argentina & 0.462 & 0.248 & 0.146 & 0.057 & 0.046 & 0.023 & 0.019 \\
Australia & 0.499 & 0.230 & 0.149 & 0.059 & 0.032 & 0.016 & 0.014 \\
Canada & 0.437 & 0.282 & 0.146 & 0.057 & 0.042 & 0.011 & 0.024 \\
Czech Republic & 0.440 & 0.259 & 0.141 & 0.073 & 0.038 & 0.025 & 0.023 \\
Germany & 0.534 & 0.221 & 0.129 & 0.056 & 0.034 & 0.009 & 0.018 \\
Hong Kong & 0.503 & 0.222 & 0.147 & 0.063 & 0.031 & 0.017 & 0.018 \\
Ireland & 0.423 & 0.271 & 0.146 & 0.074 & 0.033 & 0.025 & 0.029 \\
Japan & 0.530 & 0.246 & 0.120 & 0.052 & 0.027 & 0.012 & 0.013 \\
Mexico & 0.445 & 0.263 & 0.140 & 0.075 & 0.034 & 0.022 & 0.021 \\
Portugal & 0.413 & 0.278 & 0.131 & 0.081 & 0.036 & 0.023 & 0.038 \\
Russia & 0.438 & 0.261 & 0.139 & 0.068 & 0.036 & 0.031 & 0.027 \\
Singapore & 0.401 & 0.268 & 0.179 & 0.075 & 0.036 & 0.015 & 0.027 \\
The UK & 0.499 & 0.273 & 0.124 & 0.057 & 0.027 & 0.009 & 0.011 \\
The US & 0.500 & 0.264 & 0.129 & 0.052 & 0.032 & 0.010 & 0.013 \\
fGn with $H=0.3$ & 0.593 & 0.251 & 0.096 & 0.036 & 0.014 & 0.005 & 0.003 \\
fGn with $H=0.4$ & 0.552 & 0.252 & 0.111 & 0.049 & 0.021 & 0.009 & 0.007 \\
fGn with $H=0.5$ & 0.500 & 0.250 & 0.125 & 0.063 & 0.031 & 0.016 & 0.016 \\
fGn with $H=0.6$ & 0.440 & 0.242 & 0.134 & 0.080 & 0.044 & 0.025 & 0.035 \\
fGn with $H=0.7$ & 0.365 & 0.220 & 0.143 & 0.092 & 0.063 & 0.041 & 0.076 \\
\hline
\end{tabular}

Table 2. The stock market indices.

\begin{tabular}{cccc}
\hline Market & Index & Time period & No. observations \\
\hline Argentina & MerVal & $1 / 1997-12 / 2004$ & 1971 \\
Australia & All Ordinaries & $1 / 1993-12 / 2004$ & 3042 \\
Canada & S \& P/TSX Composite & $1 / 1993-12 / 2004$ & 3020 \\
Czech Republic & PX50 & $1 / 1998-12 / 2004$ & 1739 \\
Germany & DAX & $1 / 1993-12 / 2004$ & 3027 \\
Hong Kong & Hang Sang & $1 / 1993-12 / 2004$ & 2968 \\
Ireland & ISEQ Overall & $1 / 1993-12 / 2004$ & 3012 \\
Japan & Nikkei 225 & $1 / 1993-12 / 2004$ & 2955 \\
Mexico & IPC & $1 / 1993-12 / 2004$ & 3000 \\
Portugal & PSI20 & $1 / 1993-12 / 2004$ & 2977 \\
Russia & Moscow Time & $1 / 1995-12 / 2004$ & 2460 \\
Singapore & Straits Times & $1 / 1993-12 / 2004$ & 3016 \\
The UK & FTSE100 & $1 / 1993-12 / 2004$ & 3031 \\
The US & DJI & $1 / 1993-12 / 2004$ & 3024 \\
\hline
\end{tabular}

1) The linear fit of the Argentinean market behaves similarly to fGn with $H(\tau, 1)=0.6$ (persistent), indicating that it is essentially an emerging market (Similarly, this can be shown for the Czech, Irish, Mexican, Portuguese, Russian markets). 


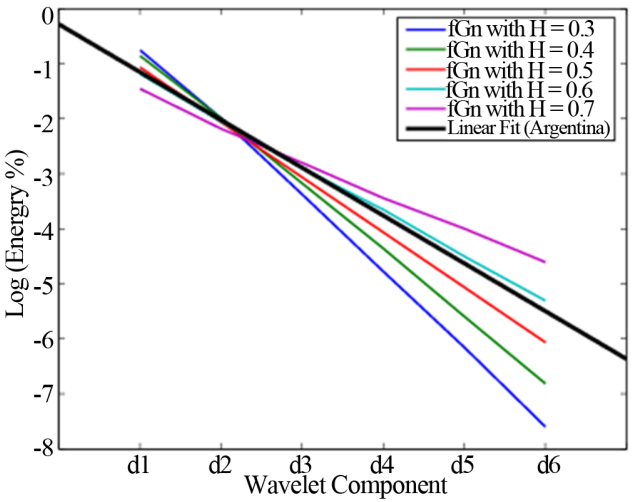

(a)

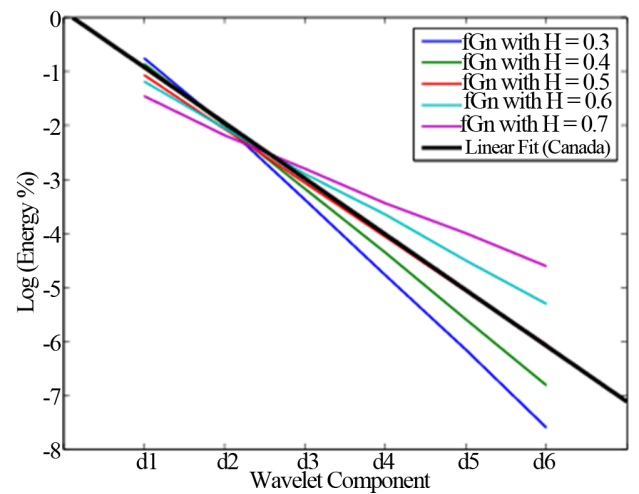

(c)

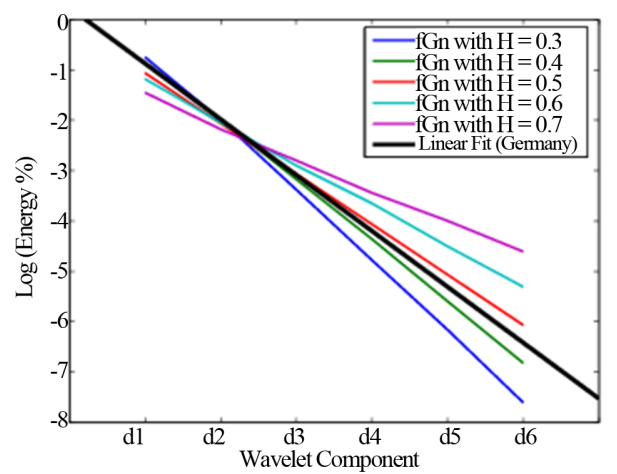

(e)

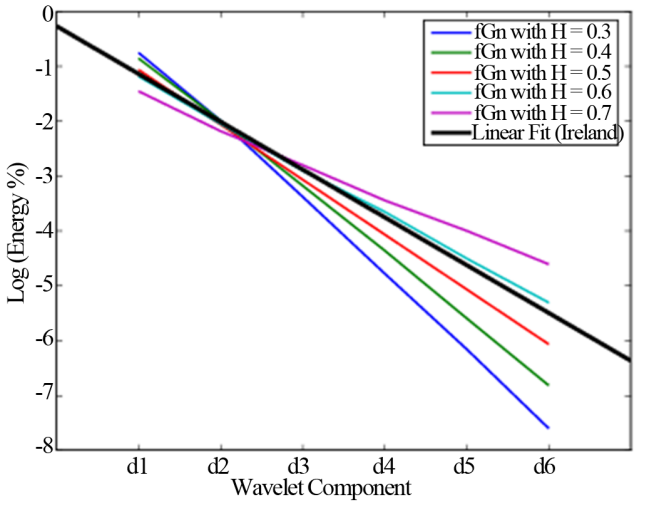

(g)

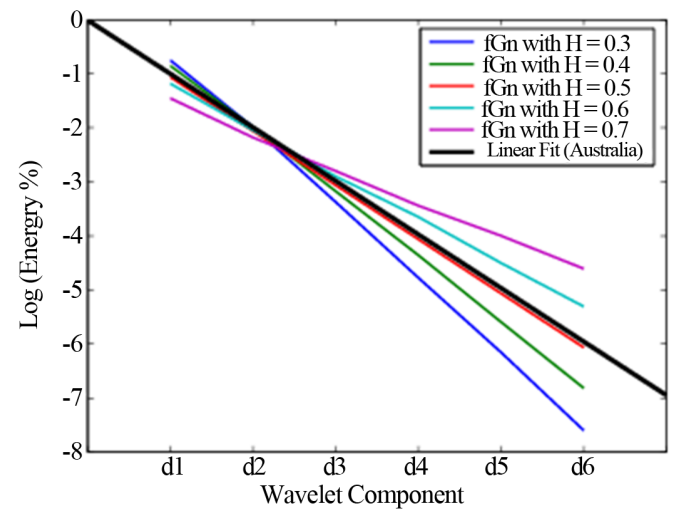

(b)

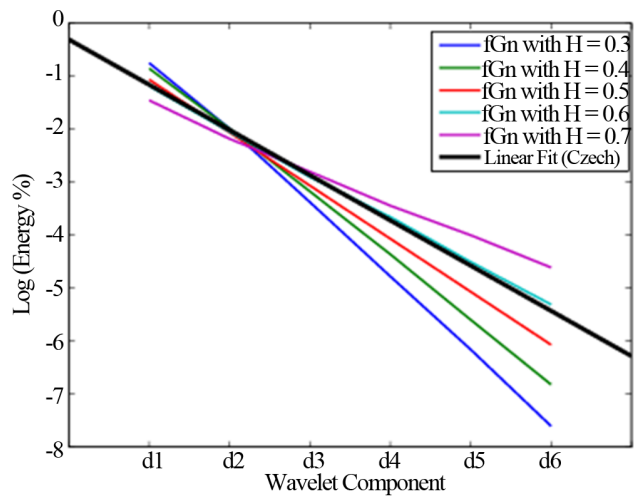

(d)

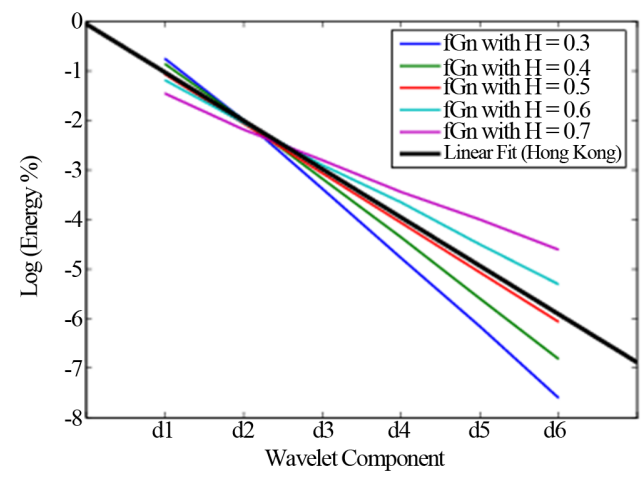

(f)

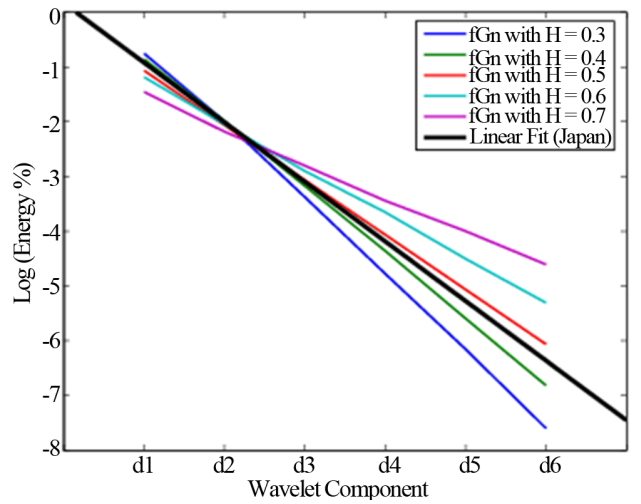

(h) 


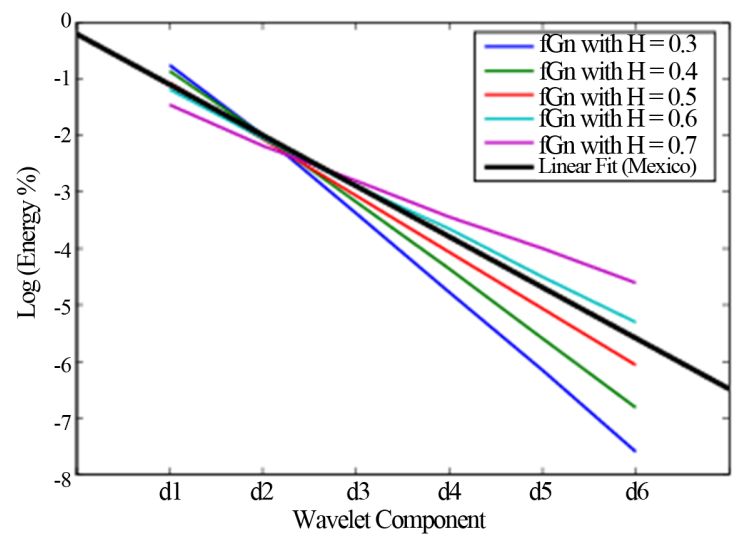

(i)

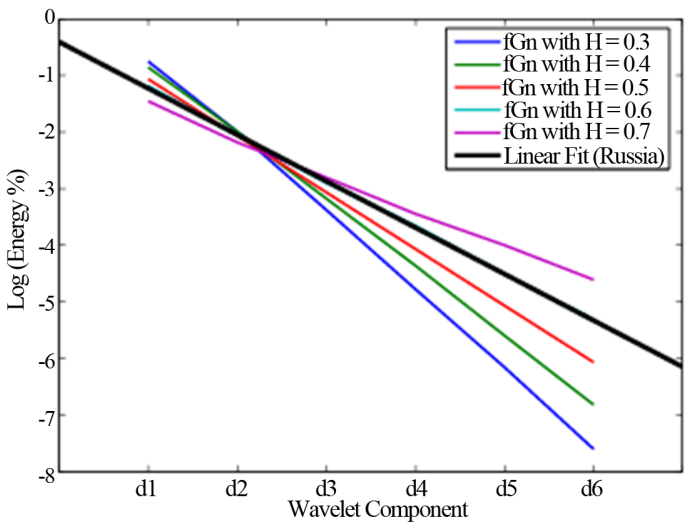

(k)

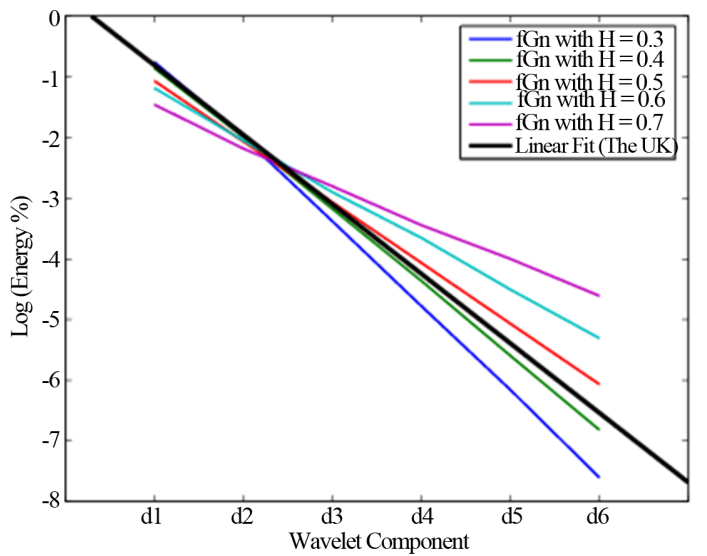

(m)

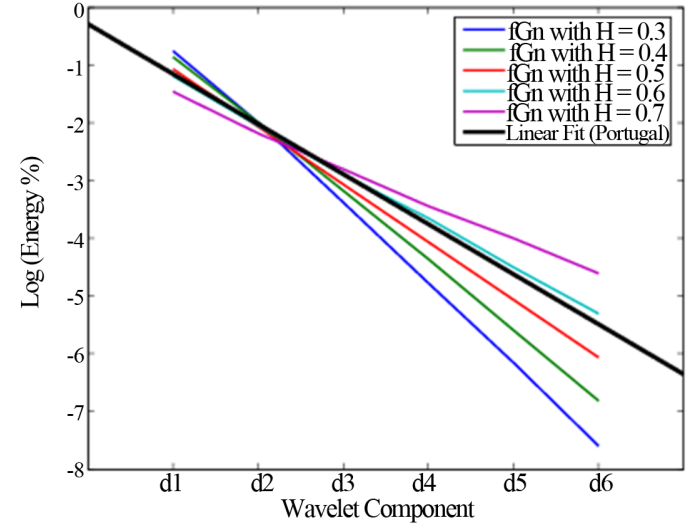

(j)

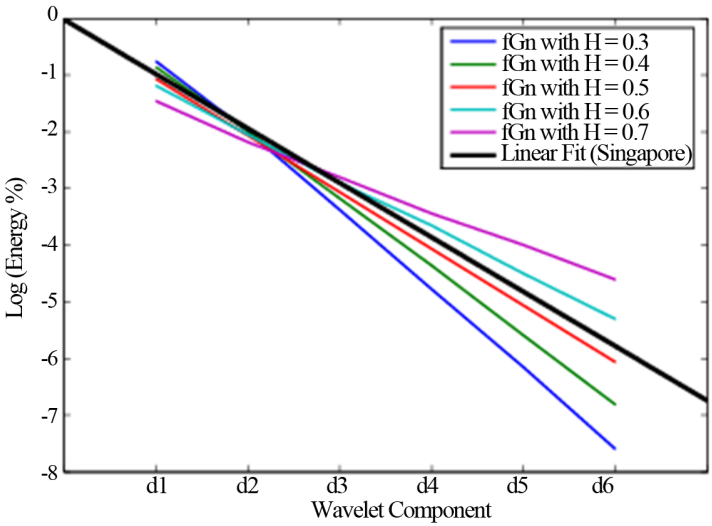

(l)

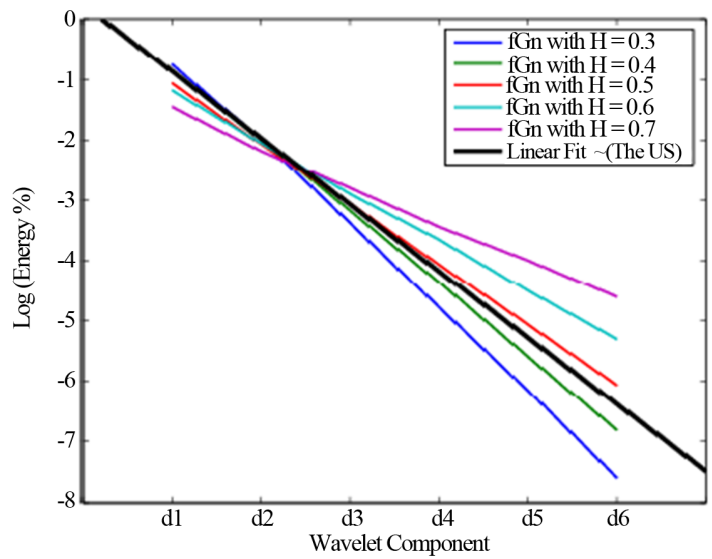

(n)

Figure 1. The logarithm to base two of the energy percentages $\left(\log _{2}(\right.$ energy\%)). (a) Argentinean market. (b) Australian market. (c) Canadian market. (d) Czech market. (e) German market. (f) Hong Kong market. (g) Irish market. (h) Japanese market. (i) Mexican market. (j) Portuguese market. (k) Russian market. (l) Singapore market. (m) UK market. (n) US market.

2) The Australian market behaves like to fGn with $H \cong 0.5$ (or Gaussian noise), meaning that this market has graduated from the emerging group, but is not yet in the mature one (Similarly, Canada, Hong Kong and Singapore).

3) However, the UK market fit is close to that for fGn with $H<0.5$ (anti-persistent), indicating that it is a mature market, (similar behaviour is demonstrated for the German, Japanese and US markets).

It is widely known that emerging and mature stock markets behave in a persistent (or long memory) and anti- 
Table 3. Classical and our new classifications of stock market.

\begin{tabular}{cccc}
\hline Market & Index & Classical classification & Our classification \\
\hline Argentina & MerVal & Emerging & Emerging \\
Australia & All Ordinaries & Mature & Intermediate \\
Canada & S\&P/TSX Composite & Mature & Intermediate \\
Czech Republic & PX50 & Emerging & Emerging \\
Germany & DAX & Mature & Mature \\
Hong Kong & Hang Sang & Mature & Intermediate \\
Ireland & ISEQ Overall & Emerging & Emerging \\
Japan & Nikkei 225 & Mature & Mature \\
Mexico & IPC & Emerging & Emerging \\
Portugal & PSI20 & Emerging & Emerging \\
Russia & Moscow Time & Emerging & Emerging \\
Singapore & Straits Times & Emerging & Intermediate \\
The UK & FTSE100 & Mature & Mature \\
The US & DJI & Mature & Mature \\
\hline
\end{tabular}

persistent (or intermediate memory) manner respectively. However, our classifier indicated that there are other stock markets which lie outside these two groups and show short memory (or independent) behaviour. On this basis, we suggest that stock markets should be classified into three different classes or categories, reflecting common characteristic and implying that stock markets bi-classification is inadequate (Table 3).

\section{Conclusions}

A novel wavelet-based algorithm was applied to the return series of fourteen stock market indices and the results show that stock market characterisation behaviour (persistent, anti-persistent or short-term) may be determined according to the Hurst exponent associated with its degree of development. This degree of development may be rooted in a number of factors, e.g. market size, liquidity, volatility, global integration, etc. The approach of using fGn and DWT, in particular, allows us to explore the overall behaviour of these markets.

Summarising the findings of this preliminary study, it appears therefore that wavelet-based approaches, in regard to stock market evolution/re-classification, show considerable potential. The implications of our method and the analysis performed are that stock markets can be grouped into three categories designated here as emerging, intermediate (or young mature) and mature (or fully mature) markets. The properties associated with this new classification need to be examined in further detail, but it does seem clear that class 2 is a particularly interesting one due to the possibility of being a new "attractive" stock market type. These markets seem to behave as Gaussian noise (or a pure random walk) indicating that they are less risky on average than emerging markets but also provide more returns than mature ones. Finally, added value applies in relation to grouping of the stocks themselves, in terms of the market composition (in [10], for example, the authors found that the new clustering, introduced in January 2006, of stocks from the FTSE100 index is more rational than the previous one because stocks from the same group (or sector) are more closely connected than those for the earlier case). This requirement to reclassify is due to rapid changes in individual stocks' behaviour.

\section{REFERENCES}

[1] A. Demirgüc-Kunt and R. Levine, "Stock Market Development and Financial Intermediaries,” The World Bank, Policy Research Working Paper No. 1462, 1995.

[2] T. Di Matteo, T. Aste and M. M. Dacorogna, "Scaling Behaviours in Differently Developed Markets," Physica A, Vol. 324, 2003, pp. 183-188. http://dx.doi.org/10.1016/S0378-4371(02)01996-9

[3] T. Di Matteo, T. Aste and M. M. Dacorogna, "Long-Term Memories of Developed and Emerging Markets: Using the Scaling Analysis to Characterise Their Stage of Development,” Journal of Banking and Finance, Vol. 29, No. 4, 2005, pp. 827-851. 
http://dx.doi.org/10.1016/j.jbankfin.2004.08.004

[4] A. Sharkasi, H. J. Ruskin, M. Crane and J. A. M. Matos, "The Reaction of Stock Markets to Crashes and Events: A Comparison Study between Emerging and Mature Markets Using Wavelet Transforms,” Physica A, Vol. 368, No. 2, 2008, pp. 39103915.

[5] J. A. M. Matos, S. M. Gama, A. Sharkasi, H. J. Ruskin and M. Crane, “Time and Scale Hurst Exponent Analysis for Financial Markets,” Physica A, Vol. 387, No. 15, 2008, pp. 3910-3915. http://dx.doi.org/10.1016/j.physa.2008.01.060

[6] R. Fuss, “The Financial Characteristics between Emerging and Developed Equity Markets,” Proceedings of Policy Modelling International Conference, Brussels, July 2002.

[7] S. Patel and A. Sarkar, “Stock Market Crises in Developed and Emerging Stock Markets,” Federal Reserve Bank of New York, Research Paper No. 9809, 1998.

[8] R. Salomons and H. Grootveld, “The Equity Risk Premium: Emerging versus Developed Markets,” University Groningen SOM Working Paper No. 02E45, 2002.

[9] P. D. Wooldridge, D. Domanski and A. Cobau, “Changing Links between Mature and Emerging Financial Markets,” BIS Quarterly Review, 2002, pp. 45-54.

[10] R. Coelho, S. Hutzler, P. Repetowicz and P. Richmond, “Sector Analysis for a FTSE Portfolio of Stocks,” Physica A, Vol. 373, 2006, pp. 615-626. http://dx.doi.org/10.1016/j.physa.2006.02.050 